\title{
VALUATION OF EUROPEAN STYLE COMPOUND OPTION WRITTEN ON EUROPEAN STYLE CURRENCY AND POWER OPTIONS
}

\author{
JAVED HUSSAIN* \\ Sukkur IBA University, Pakistan \\ *Corresponding author: javed.brohi@iba-suk.edu.pk
}

\begin{abstract}
The aim of the paper is paper is twofold. Firstly, we will derive an explicit closed formula for pricing the compound call option contingent upon a currency call option. Secondly, we will develop a pricing formula for the compound call option contingent upon the power call option.
\end{abstract}

\section{INTRODUCTION}

A party with the long position in a currency option has the right ( but not obligation) to trade (buy/sell) a predetermined amount of the Foreign currency at a fixed predetermined price (strike pricier in domestic currency) on the fixed agreed date (maturity/expiry). This option can be found in both American and EU styles. Certainly, the investor would prefer to enter into the long position currency call if he/she expects that the foreign currency is going to go high, on contrast, if the investor expects that the value of a foreign currency is going decline he/she will prefer to enter input option. There are numerous reasons that why currencies fluctuate, obviously with the normal fluctuations in currency can be explained by demand-supply principle, but a case of political uncertainty, financial crises, and natural disasters (such COVID-19 pandemic) the currencies fluctuated gigantically. This makes the currency option a major tool for the management of risk associated with the evolution of currencies. In [15, 1979], introduced the currency option but they could not give an explicit formula for the premium of the currency option. In the Black-Scholes framework,

Received August 22 $2^{\text {nd }}, 2020$; accepted September $14^{\text {th }}, 2020$; published October $7^{\text {th }}, 2020$.

2010 Mathematics Subject Classification. 91G20.

Key words and phrases. financial derivatives; option pricing; compound option; currency option; power option.

(C)2020 Authors retain the copyrights of their papers, and all open access articles are distributed under the terms of the Creative Commons Attribution License. 
an explicit formula for pricing currency option was studied by Grabbe in [16, 1983], where the underlying currency option is driven by geometric Brownian motion. They also deduced that American style currency options are more expensive than that of their EU counterpart. Amin and Jarrow in [17, 1991], studied and priced the EU style currency option in the Heath-Jarrow Morton (HJM) framework, under a strong assumption of constancy of volatility. To deal with the situations where the foreign currency fluctuates with high magnitude i.e. volatility several attempts have been made under the assumption that foreign currency follows a stochastic differential driven various version of sophisticated jump-diffusion processes, for instance, see Hung [18, 2007], Li, Peng [19, 2013], Ming [21, 2019] and David Liu [20, 20].

Power option is a type of polynomial option in which payoff depends on the basic resources (underlying assets) raised to a certain power. It is structured to allow the buyer (holder) to take a securitized view of a particular asset or its volatility. They have been created to modify the patterns of plain vanilla options. The payoff of a power option is the difference between the underlying asset price at maturity raised to some strictly positive power. For power call options, Payoff is $\max \left(S^{i}-X, 0\right)$ while for power put options, payoff is $\max \left(X-S^{i}, 0\right)$ where $i$ is some positive integer power $i>0$.

In $[2,1973]$ Black, F. and Scholes, M., suggested that power options can be beneficial for an investor in bringing more premium than an ordinary option which is often the reason of a sharp-sighted investor's attraction. In [3, 2006], Macovshi has studied power option and shown that polynomial option can be priced through expressing it as a combination of different power options with adapted strikes.

In Zhang [4, 2016], the pricing formula for the power option is driven for Liu's uncertain stock model under the assumption that the underlying stock price follows some probabilistic (Stochastic) differential equation. In Dastranj [5, 2017] Option pricing under Double Heston model and Double Heston with three jumps are discussed, concluding that in comparison to all other options, Power Option produces more premium income under the Double Heston model. In Saberi [6, 2018] power option pricing is derived using time-fractional PDE for the dynamics of underlying asset price that follows a regime-switching model in which the underlying risky asset mainly depends on a continuous-time hidden Markov chain process. They've further produced an exact solution for their proposed power option. In Dastranj [7, 2020] a hypothetical option pricing based on fractional Heston model is discussed for Iran's gold market. Using Fast Fourier Transformation, the pricing of the power option has been driven. The analysis brings forward that in most of the three months periods the power option prevented from creating arbitrage opportunity while related to 2018 , two periods have been observed where power option created an arbitrage opportunity due to high volatilities.

A compound option also called the Split-Fee option is an option over another option that enables a holder with the right to purchase or sell another option. One can have four possible types of compound options, 
namely' call on call, call on put, put on put or put on call each with two dates of expiration and two strike prices. The usage of compound options mainly involves currency or fixed income markets.

In Geske $[8,1977]$ risky coupon bond problem is tackled using the compound option valuing technique. In Geske $[9,1977]$ compound option is computed by defining a call option on a stock which is itself an option on the firm assets. This valuation result was extended by Hull $[10,2003]$ to a compound option with underlying assets paying a known dividend return. Elettra [11, 2003] considered the time-dependent volatility and interest rate for deriving the pricing model which is a generalized and more realistic version of Geske [9, 1979]. Sequential exchange options in Carr [12] are priced using the compound options. American type compound options are priced for stochastic interest rates and stochastic volatility in Chiarella [13, 2013]. In Li [14, 2020] analytic pricing formula for compound options is driven in terms of Fourier integral of the characteristic function. The obtained formula was used along with the FFT algorithm to calculate the compound option's price across the entire spectrum of the exercise price.

Section 1 is presenting the introduction based on the available literature. Section 2 introduces Foreign Currency Options, gives expressions for their payoff and later explains Compound Options written on Currency Options using Feynman-Kac Formula. In section 3 European Power Option is explained followed by expressions for its payoff and then European Compound Options over Power call Option are derived in detail in detail.

\section{Compound Option Written On Foreign Currency Option}

Before moving towards the option pricing we will would like to mention a result that we will use frequently throughout the paper.

Theorem 2.1. (Identity for Bivariate Standard Normal Distribution) [23] Let $X \sim \mathscr{N}(0,1)$ and $Y \sim$ $\mathscr{N}(0,1)$ be jointly normally distributed with correlation coefficient $\rho \in(-1,1)$, then joint cumulative distribution function can be written as following

$$
\boldsymbol{\Phi}\left(\alpha, \beta, \rho_{x y}\right)=\int_{-\infty}^{\alpha} n(x) \Phi\left(\frac{\beta-\rho x}{\sqrt{1-\rho^{2}}}\right) d x
$$

Here $\Phi$ denotes cumulative distribution of standard normal random, $n(x)$ denotes the probability distribution function of standard normal random variable and $\mathbf{\Phi}$ denotes the bivariate cumulative distribution function.

2.1. Foreign Currency Option. [10] A foreign currency option is like a European option give the right to option holder to buy or to sell one unit of foreign currency at time $T$ for the price $K$ at predetermined exchange rate $K$. The difference between foreign and European options is that in currency options risk-free rate is replaced by foreign interest rate. The payoff of the foreign currency option at time $T$ for the strike price $K$ is given by:

$$
C_{c u r}(T, K)=\max \left[\left(S_{T}-K\right), 0\right] .
$$


The derivation of the Currency option is the same as the Black-Scholes methodology under the following assumption. The Foreign Currency option which is underlying assets follows the Geometric Brownian motion. The foreign and domestic interest rates are constant during the whole life of the contract. Hence the pricing formula for Currency call are,

$$
C_{\text {cur }}\left(t, S_{t}, K\right)=S_{t} e^{-r_{f}(T-t)} \Phi\left(d_{2}\right)-K_{2} e^{-r_{D}(T-t)} N\left(d_{1}\right)
$$

where,

$$
d_{1}=\frac{\ln \left(\frac{S_{t}}{K}\right)+\left(r_{D}-r_{f}+\frac{\sigma^{2}}{2}\right)(T-t)}{\sigma \sqrt{T-t}} \quad d_{2}=\frac{\ln \left(\frac{S_{t}}{K}\right)-\left(r_{D}-r_{f}-\frac{\sigma^{2}}{2}\right)(T-t)}{\sigma \sqrt{T-t}} .
$$

Where $r_{f}$ is the foreign interest rate and $r_{D}$ is the domestic interest rate, the value of Call Option will increase for currency if domestic interest rate increases and the value will down if the domestic interest rate will fall.

\subsection{Compound Option written on Currency Option.}

Theorem 2.2. Let $\left\{W_{t}: t \geq 0\right\}$ be a $\mathbb{P}$-standard Brownian motion on the probability space $(\Omega, \mathcal{F}, \mathbb{P})$ and let $S_{t}$, the price of unit of foreign currency, follow a GBM with the following SDE

$$
d S_{t}=\mu S_{t} d t+\sigma S_{t} d W_{t}
$$

where $\mu$ is the drift parameter and $\sigma$ is the volatility parameter. In addition, we let $r$ be the risk-free interest rate. Then the price of compound call option, with strike $K_{1}$ and maturity $T_{1}$, contingent upon a currency call option, with strike $K_{2}$ and maturity $T_{2}$, is given by,

$$
C_{C_{c u r}}\left(t, S_{t}, K_{1}, K_{2}, T_{1}, T_{2}\right)=\mathbf{\Phi}\left(w_{1}, w_{3} ; \rho\right)-K_{2} e^{-r_{D}\left(T_{2}-t\right)} \mathbf{\Phi}\left(w_{2}, w_{4} ; \rho\right)-K_{1} e^{-r_{D}\left(T_{1}-t\right)} \Phi\left(w_{1}\right)
$$

where,

$$
\begin{gathered}
\rho=\frac{\sqrt{T_{1}-t}}{\sqrt{T_{2}-t}}, w_{1}=\frac{\ln \left(\frac{K_{1}}{S_{t}}\right)-\left(r_{D}-\frac{\sigma^{2}}{2}\right)\left(T_{1}-t\right)}{\sigma \sqrt{T_{1}-t}}, w_{2}=\frac{\ln \left(\frac{K_{1}}{S_{t}}\right)-\left(r_{D}+\frac{\sigma^{2}}{2}\right)\left(T_{1}-t\right)}{\sigma \sqrt{T_{1}-t}} \\
w_{3}=\frac{\ln \left(\frac{S_{t}}{K_{2}}\right)+\left(r_{D}-\frac{\sigma^{2}}{2}\right)\left(T_{2}-t\right)-r_{f}\left(T_{2}-T_{1}\right)}{\sigma \sqrt{T_{2}-T_{1}}}, \\
w_{4}=\frac{\ln \left(\frac{S_{t}}{K_{2}}\right)+\left(r_{D}+\frac{\sigma^{2}}{2}\right)\left(T_{2}-t\right)-r_{f}\left(T_{2}-T_{1}\right)}{\sigma \sqrt{T_{2}-T_{1}}} .
\end{gathered}
$$

In this pricing formula, $\Phi$ represents distribution function of standard normal distribution and $\mathbf{\Phi}$ represents the bivariate cumulative distribution function of standard normal distribution and $\rho$ is the correlation. 
Proof. We will start by providing an explicit Black-Scholes price (cf. [16]) of the underlying currency option with maturity $T_{2}$ and strike $K_{2}$, with inception at $T_{1}$, can be given as,

$$
C_{c u r}=S_{T_{1}} e^{-r_{f}\left(T_{2}-T_{1}\right)} \Phi\left(d_{2}\right)-K_{2} e^{-r_{D}\left(T_{2}-T_{1}\right)} \Phi\left(d_{1}\right) .
$$

Here

$$
d_{1}=\frac{\ln \left(\frac{S_{T_{1}}}{K}\right)+\left(r_{D}-r_{f}+\frac{\sigma^{2}}{2}\right)\left(T_{2}-T_{1}\right)}{\sigma \sqrt{T_{2}-T_{1}}}, \quad d_{2}=\frac{\ln \left(\frac{S_{T_{1}}}{K}\right)-\left(r_{D}-r_{f}-\frac{\sigma^{2}}{2}\right)\left(T_{2}-T_{1}\right)}{\sigma \sqrt{T_{2}-T_{1}}} .
$$

Consider the risk-neutral measure $\mathbb{Q}$, such that $S_{t}$ follows

$$
d S_{t}=\left(r_{D}-r_{f}\right) S_{t} d t+\sigma d W_{t}^{Q}
$$

where $W_{t}^{\mathbb{Q}}=W_{t}+\left(\frac{\mu-\left(r_{D}-r_{f}\right)}{\sigma}\right) t$ is a $\mathbb{Q}$-standard Wiener process. Indeed for $T>t, \log$ returns $\ln \left(\frac{S_{T}}{S_{t}}\right)$ follows $\mathcal{N}\left[\left(\left(r_{D}-r_{f}\right)-\frac{\sigma^{2}}{2}\right)(T-t), \sigma^{2}(T-t)\right]$ with conditional density function,

$$
n\left(S_{T} \mid S_{t}\right)=\frac{1}{S_{T} \sigma \sqrt{2 \pi(T-t)}} e^{-\frac{1}{2}\left(\frac{\ln \left(\frac{S_{T}}{S_{t}}\right)-\left(\left(r_{D}-r_{f}\right)-\frac{\sigma^{2}}{2}\right)(T-t)}{\sigma \sqrt{T-t}}\right)^{2}} .
$$

Next using the Feynman-Kac formula [22, Theorem 4.33], the price of EU. compound option, contingent upon currency option, under risk-neutral probability measure $\mathbb{Q}$, at time $t \leq T_{1} \leq T_{2}$, can be given as, $C_{C_{c u r}}\left(t, S_{t}, K_{1}, K_{2}, T_{1}, T_{2}\right)$

$$
\begin{aligned}
= & \left.e^{-r_{D}\left(T_{1}-t\right)} \mathbb{E}^{\mathbb{Q}}\left[C_{c u r}\left(S_{T_{1}}, T_{1} ; K_{2}, T_{2}\right)-K_{1}\right)^{+} \mid \mathcal{F}_{t}\right] \\
= & e^{-r_{D}\left(T_{1}-t\right)} \mathbb{E}_{\mathbb{Q}}\left[\left(S_{T_{1}} e^{-r_{f}\left(T_{2}-T_{1}\right)} \Phi\left(d_{2}\right)-K_{2} e^{-r_{D}\left(T_{2}-T_{1}\right)} \Phi\left(d_{1}\right)-K_{1}\right)^{+} \mid \mathcal{F}_{t}\right] \\
= & e^{-r_{D}\left(T_{1}-t\right)} \int_{0}^{\infty}\left[\left(S_{T_{1}} e^{-r_{f}\left(T_{2}-T_{1}\right)} \Phi\left(d_{2}\right)-K_{2} e^{-r_{D}\left(T_{2}-T_{1}\right)} \Phi\left(d_{1}\right)\right)-K_{1}\right]^{+} \\
& \times n\left(S_{T_{1}} \mid S_{t}\right) d S_{T_{1}} .
\end{aligned}
$$

Let $S^{*}$ be price of unit of foreign currency such that, the compound option is at the money i.e. $C\left(S^{*}, T_{1} ; K_{2}, T_{2}\right)=K_{1}$, and using this equation and any suitable method (say Newton-Raphson) an approximate value of $S^{*}$ can be computed. Now,

$C_{C_{c u r}}\left(t, S_{t}, K_{1}, K_{2}, T_{1}, T_{2}\right)$

$$
\begin{aligned}
= & e^{-r_{D}\left(T_{1}-t\right)} \int_{S^{*}}^{\infty}\left(\left(S_{T_{1}} e^{-r_{f}\left(T_{2}-T_{1}\right)} \Phi\left(d_{2}\right)-K_{2} e^{-r_{D}\left(T_{2}-T_{1}\right)} \Phi\left(d_{1}\right)\right)-K_{1}\right) n\left(S_{T_{1}} \mid S_{t}\right) d S_{T_{1}}, \\
= & e^{-r_{D}\left(T_{1}-t\right)} \int_{S^{*}}^{\infty}\left(S_{T_{1}} e^{-r_{f}\left(T_{2}-T_{1}\right)} \Phi\left(d_{2}\right)\right) n\left(S_{T_{1}} \mid S_{t}\right) d S_{T_{1}} \\
& -K_{2} e^{-r_{D}\left(T_{2}-t\right)} \int_{S^{*}}^{\infty} \Phi\left(d_{1}\right) n\left(S_{T_{1}} \mid S_{t}\right) d S_{T_{1}}-K_{1} e^{-r_{D}\left(T_{1}-t\right)} \int_{S^{*}}^{\infty} n\left(S_{T_{1}} \mid S_{t}\right) d S_{T_{1}} \\
=: & I_{1}-I_{2}-I_{3} .
\end{aligned}
$$


Let us begin by computing the integral $I_{1}$,

$$
\begin{aligned}
I_{1} & =e^{-r_{D}\left(T_{1}-t\right)} \int_{S^{*}}^{\infty}\left(S_{T_{1}} e^{-r_{f}\left(T_{2}-T_{1}\right)} \Phi\left(d_{2}\right)\right) n\left(S_{T_{1}} \mid S_{t}\right) d S_{T_{1}}, \\
& =e^{-r_{D}\left(T_{1}-t\right)} \int_{S^{*}}^{\infty}\left(S_{T_{1}} e^{-r_{f}\left(T_{2}-T_{1}\right)} \Phi\left(d_{2}\right)\right) n\left(S_{T_{1}} \mid S_{t}\right) d S_{T_{1}} .
\end{aligned}
$$

Now the compound option will be in the money and hence will be exercised iff $S_{T_{1}}>$ $S^{*} \quad$ iff $S_{t} e^{\left(\left(r_{D}-r_{f}\right)\left(T_{1}-t\right)+\sigma\left(W_{T_{1}}^{\Theta}-W_{t}^{\bigotimes}\right)\right)}>S^{*}$ iff $S_{t} e^{\left(\left(r_{D}-r_{f}-\frac{\sigma^{2}}{2}\right)\left(T_{1}-t\right)+\sigma \sqrt{T_{1}-t} x\right)}>S^{*}$ iff $x>$ $\frac{\ln \left(\frac{S^{*}}{S_{t}}\right)-\left(r_{D}-r_{f}-\frac{\sigma^{2}}{2}\right)\left(T_{1}-t\right)}{\sigma \sqrt{T_{1}-t}}=: w_{1}$. Hence we rewrite $I_{1}$ as,

$$
\begin{aligned}
I_{1}= & e^{-r_{D}\left(T_{1}-t\right)} S_{t} e^{\left(r_{D}-r_{f}-\frac{\sigma^{2}}{2}\right)\left(T_{1}-t\right)-r_{f}\left(T_{2}-T_{1}\right)} \times \\
& \int_{w_{1}}^{\infty} \Phi\left(\frac{\ln \left(\frac{S_{t}}{K_{2}}\right)+\left(r_{D}-r_{f}+\frac{\sigma^{2}}{2}\right)\left(T_{2}-T_{1}\right)+\left(r_{D}-r_{f}-\frac{\sigma^{2}}{2}\right)\left(T_{1}-t\right)+x \sigma \sqrt{T_{1}-t}}{\sigma \sqrt{T_{2}-T_{1}}}\right) \\
& \left.\times \frac{e^{-\frac{\left(x^{2}-2 x \sigma \sqrt{T_{1}-t}\right)}{2}} d x}{\sqrt{2 \pi}}\right) \\
= & S_{t} e^{-r_{f}\left(T_{2}-t\right)-\frac{\sigma^{2}}{2}\left(T_{1}-t\right)} e^{\frac{\sigma^{2}}{2}\left(T_{1}-t\right)} \times \\
& \int_{w_{1}}^{\infty} \Phi\left(\frac{\ln \left(\frac{S_{t}}{K_{2}}\right)+\left(r_{D}-r_{f}+\frac{\sigma^{2}}{2}\right)\left(T_{2}-T_{1}\right)+\left(r_{D}-r_{f}-\frac{\sigma^{2}}{2}\right)\left(T_{1}-t\right)+x \sigma \sqrt{T_{1}-t}}{\sigma \sqrt{T_{2}-T_{1}}}\right) \\
& \times \frac{e^{-\frac{\left(x-\sigma \sqrt{T_{1}-t}\right)^{2}}{2}} d x}{\sqrt{2 \pi}} d x
\end{aligned}
$$

To simplify the above integral, let using substitution $u=-\left(x-\sigma \sqrt{T_{1}-t}\right)$, the integral simplifies to,

$$
I_{1}=S_{t} e^{-r_{f}\left(T_{2}-t\right)} \int_{-\infty}^{w_{2}} \Phi\left(\frac{\ln \left(\frac{S_{t}}{K_{2}}\right)+\left(r_{D}-r_{f}+\frac{\sigma^{2}}{2}\right)\left(T_{2}-t\right)-u \sigma \sqrt{T_{1}-t}}{\sigma \sqrt{T_{2}-T_{1}}}\right) \frac{e^{-\frac{u^{2}}{2}}}{\sqrt{2 \pi}} d u
$$

where $w_{2}=\sigma \sqrt{T_{1}-t}-w_{1}$. If we set $\rho=\sqrt{\frac{T_{1}-t}{T_{2}-t}}$ and $w_{4}=\frac{\ln \left(\frac{S_{t}}{K_{2}}\right)+\left(r_{D}-r_{f}+\frac{\sigma^{2}}{2}\right)\left(T_{2}-t\right)}{\sigma \sqrt{T_{2}-t}}$ along with using Bivariate identity (2.1) the last integral can be written as,

$$
I_{1}=S_{t} e^{-r_{f}\left(T_{2}-t\right)} \int_{-\infty}^{\psi_{2}} \Phi\left(\frac{w_{4}-\rho u}{\sqrt{1-\rho^{2}}}\right) \frac{e^{-\frac{u^{2}}{2}}}{\sqrt{2 \pi}} d u=e^{-r_{f}\left(T_{2}-t\right)} \mathbf{\Phi}\left(w_{2}, w_{4} ; \rho\right) .
$$

Now let us compute the second integral $I_{2}$,

$$
I_{2}=K_{2} e^{-r_{D}\left(T_{2}-t\right)} \int_{S^{*}}^{\infty} \Phi\left(d_{1}\right) n\left(S_{T_{1}} \mid S_{t}\right) d S_{T_{1}}
$$

By arguing same as previously, the compound option will be in the money and hence will be exercised iff $S_{T_{1}}>S^{*}$ iff $S_{t} e^{\left(\left(r_{D}-r_{f}\right)\left(T_{1}-t\right)+\sigma\left(W_{T_{1}}^{\complement}-W_{t}^{\complement}\right)\right)}>S^{*}$ iff $S_{t} e^{\left(\left(r_{D}-r_{f}-\frac{\sigma^{2}}{2}\right)\left(T_{1}-t\right)+\sigma \sqrt{T_{1}-t} x\right)}>S^{*}$ iff $x>-\frac{\ln \left(\frac{S_{t}}{S^{*}}\right)-\left(r_{D}-r_{f}-\frac{\sigma^{2}}{2}\right)\left(T_{1}-t\right)}{\sigma \sqrt{T_{1}-t}}=-w_{1}$. Hence we rewrite $I_{2}$ as,

$$
I_{2}=K_{2} e^{-r_{D}\left(T_{1}-t\right)} \int_{-w_{1}}^{\infty} \Phi\left(\frac{\ln \left(\frac{S_{t}}{K_{2}}\right)+\left(r_{D}-r_{f}-\frac{\sigma^{2}}{2}\right)\left(T_{2}-t\right)+x \sigma \sqrt{T_{1}-t}}{\sigma \sqrt{T_{2}-T_{1}}}\right) \frac{e^{-\frac{x^{2}}{2}}}{\sqrt{2 \pi}} d x .
$$


Using the substitution, $u=-x$, the above integral can be simplified to,

$$
I_{2}=-K_{2} e^{-r_{D}\left(T_{1}-t\right)} \int_{w_{1}}^{-\infty} \Phi\left(\frac{\ln \left(\frac{S_{t}}{K_{2}}\right)+\left(r_{D}-r_{f}-\frac{\sigma^{2}}{2}\right)\left(T_{2}-t\right)-u \sigma \sqrt{T_{1}-t}}{\sigma \sqrt{T_{2}-T_{1}}}\right) \frac{e^{-\frac{u^{2}}{2}}}{\sqrt{2 \pi}} d u .
$$

On setting, if we set $\rho=\sqrt{\frac{T_{1}-t}{T_{2}-t}}$ and $w_{3}=\frac{\ln \left(\frac{S_{t}}{K_{2}}\right)+\left(r_{D}-r_{f}-\frac{\sigma^{2}}{2}\right)\left(T_{2}-t\right)}{\sigma \sqrt{T_{2}-t}}$ along with using Bivariate identity (2.1) the last integral can be written as,

$$
\begin{aligned}
I_{2} & =K_{2} e^{-r_{D}\left(T_{1}-t\right)} \int_{-\infty}^{w_{1}} \Phi\left(\frac{w_{3}-\rho u}{\sqrt{1-\rho^{2}}}\right) \frac{e^{-\frac{u^{2}}{2}}}{\sqrt{2 \pi}} d u, \\
& =K_{2} e^{-r_{D}\left(T_{1}-t\right)} \mathbf{\Phi}\left(w_{1}, w_{3} ; \rho\right) .
\end{aligned}
$$

Finally, its fairly easy to compute the 3 rd integral $I_{3}$, we will make use of fact $W_{T_{1}}^{\mathbb{Q}}-W_{t}^{\mathbb{Q}} \sim \mathcal{N}\left(0, T_{1}-t\right)$ we have

$$
\begin{aligned}
I_{3} & =K_{1} e^{-r_{D}\left(T_{1}-t\right)} \int_{S^{*}}^{\infty} n\left(S_{T_{1}} \mid S_{t}\right) d S_{T_{1}} \\
& =K_{1} e^{-r_{D}\left(T_{1}-t\right)} \mathbb{Q}\left(S_{T_{1}}>S^{*} \mid S_{t}\right) \\
& =K_{1} e^{-r_{D}\left(T_{1}-t\right)} \mathbb{Q}\left(S_{t} e^{\left(r_{d}-r_{f}-\frac{\sigma^{2}}{2}\right)\left(T_{1}-t\right)+\sigma\left(W_{T_{1}}^{\mathbb{Q}}-W_{t}^{\mathbb{Q}}\right)}>S^{*} \mid S_{t}\right) \\
& =K_{1} e^{-r_{D}\left(T_{1}-t\right)} \mathbb{Q}\left(S_{t} e^{\left(r_{d}-r_{f}-\frac{\sigma^{2}}{2}\right)\left(T_{1}-t\right)+\sigma \sqrt{T_{1}-t} Z}>S^{*} \mid S_{t}\right) \\
& =K_{1} e^{-r_{D}\left(T_{1}-t\right)} \mathbb{Q}\left(Z>\frac{\ln \left(\frac{S^{*}}{S_{t}}\right)-\left(r_{D}-r_{f}-\frac{\sigma^{2}}{2}\right)\left(T_{1}-t\right)}{\sigma \sqrt{T_{1}-t}}\right) \\
& =K_{1} e^{-r_{D}\left(T_{1}-t\right)} \Phi\left(w_{1}\right)
\end{aligned}
$$

Combine the integrals $I_{1}, I_{2}$ and $I_{3}$ in (2.2) we get the pricing formula for European compound Option written on Foreign Currency call option,

$$
\begin{aligned}
C_{C_{\text {cur }}}\left(t, S_{t}, K_{1}, K_{2}, T_{1}, T_{2}\right)= & e^{-r_{f}\left(T_{2}-t\right)} \mathbf{\Phi}\left(w_{2}, w_{4} ; \rho\right)-K_{2} e^{-r_{D}\left(T_{2}-t\right)} \mathbf{\Phi}\left(w_{1}, w_{3} ; \rho\right) \\
& -K_{1} e^{-r_{D}\left(T_{1}-t\right)} \Phi\left(w_{1}\right) .
\end{aligned}
$$

where

$$
\begin{array}{ll}
w_{1}=\frac{\ln \left(\frac{S^{*}}{S_{t}}\right)-\left(r_{D}-r_{f}-\frac{\sigma^{2}}{2}\right)\left(T_{1}-t\right)}{\sigma \sqrt{T_{1}-t}}, & w_{3}=\frac{\ln \left(\frac{S_{t}}{K_{2}}\right)+\left(r_{D}-r_{f}+\frac{\sigma^{2}}{2}\right)\left(T_{2}-t\right)}{\sigma \sqrt{T_{2}-t}} \\
w_{2}=\frac{\ln \left(\frac{S_{t}}{S^{*}}\right)+\left(r_{D}-r_{f}-\frac{\sigma^{2}}{2}\right)\left(T_{1}-t\right)}{\sigma \sqrt{T_{1}-t}}, & w_{4}=\frac{\ln \left(\frac{S_{t}}{K_{2}}\right)+\left(r_{D}-r_{f}-\frac{\sigma^{2}}{2}\right)\left(T_{2}-t\right)}{\sigma \sqrt{T_{2}-t}} .
\end{array}
$$

and

$$
\rho=\frac{\sqrt{T_{1}-t}}{\sqrt{T_{2}-t}}
$$

In this pricing formula, $\Phi$ represents cumulative function of standard normal distribution and $\boldsymbol{\Phi}$ represents the bivariate cumulative normal distribution and $\rho$ is the correlation. 


\section{Compound Option written on Power Option}

3.1. Power Option. [10] If an option whose payoff price is related to exponent of $\mathbb{R}^{+}$underlying assets is called European power option. this kind of option may have European power call option and European power put option whose payoff are the following,

$$
\begin{aligned}
C_{\text {Power }} & =\max \left[\left(S_{T}^{n}-K\right), 0\right], \\
P_{\text {Power }} & =\max \left[\left(K-S_{T}^{n}\right), 0\right] .
\end{aligned}
$$

The Valuation of Power option can be calculated by using Black-Scholes strategy, then the pricing formula for European call and put option is,

$$
C_{\text {Power }}\left(t, S_{t}, T, K\right)=S_{t}^{n} e^{\left[(n-1)\left(r+\frac{n \sigma^{2}}{2}\right)-n D\right](T-t)} \Phi\left(d_{1}\right)-K e^{-r(T-t)} \Phi\left(d_{2}\right),
$$

where

$$
d_{1}=\frac{\ln \left(\frac{S_{t}}{K^{\frac{1}{n}}}\right)+\left(r-D+\left(n-\frac{1}{2}\right) \sigma^{2}\right)(T-t)}{\sigma \sqrt{T-t}}, d_{2}=\frac{\ln \left(\frac{S_{t}}{K^{\frac{1}{n}}}\right)+\left(r-D-\frac{\sigma^{2}}{2}\right)(T-t)}{\sigma \sqrt{T-t}}
$$

\subsection{Compound Option written on Power option.}

Theorem 3.1. Let $\left\{W_{t}: t \geq 0\right\}$ be a $\mathbb{P}$-standard Brownian motion on the probability space $(\Omega, \mathcal{F}, \mathbb{P})$ and let $S_{t}$, the price of unit of stock, follow a GBM with the following SDE

$$
d S_{t}=(\mu-D) S_{t} d t+\sigma S_{t} d W_{t}
$$

where $\mu$ is the drift parameter and $\sigma$ is the volatility parameter. In addition, we let $r$ be the risk-free interest rate. Then the price of compound call option, with strike $K_{1}$ and maturity $T_{1}$, contingent upon a power call option, with strike $K_{2}$ and maturity $T_{2}$, is given by

$$
\begin{aligned}
C_{C_{\text {pow }}}\left(t, S_{t}, K_{1}, K_{2}, T_{1}, T_{2}\right)= & S_{t}^{n} e^{\left((n-1)\left(r+\frac{\sigma^{2}}{2}\right)-n \sigma^{2}\right)\left(T_{1}-t\right)+(n-1)\left(r+\frac{n \sigma^{2}}{2}\right)\left(T_{2}-T_{1}\right)-n D\left(T_{2}-t\right)} \\
& \times \mathbf{\Phi}\left(\psi_{2}, \psi_{4} ; \rho\right)-K_{2} e^{-r\left(T_{1}-t\right)} \boldsymbol{\Phi}\left(\psi_{1}, \psi_{3} ; \rho\right)-K_{1} e^{-r\left(T_{1}-t\right)} \Phi\left(\psi_{1}\right) .
\end{aligned}
$$


where

$$
\begin{aligned}
\psi_{1} & =\frac{\ln \left(\frac{S^{*}}{S_{t}^{n}}\right)-n\left(r-D-\frac{\sigma^{2}}{2}\right)\left(T_{1}-t\right)}{\sigma \sqrt{T_{1}-t}}, \\
\psi_{2} & =\frac{\ln \left(\frac{S_{t}^{n}}{S^{*}}\right)+n\left(r-D+\frac{\sigma^{2}}{2}\right)\left(T_{1}-t\right)}{n \sigma \sqrt{T_{1}-t}}, \\
\psi_{3} & =\frac{\ln \left(\frac{S_{t}}{K^{\frac{1}{n}}}\right)+\left(r-D+\left(n-\frac{1}{2}\right) \sigma^{2}\right)\left(T_{2}-t\right)}{n \sigma \sqrt{T_{2}-t}}, \\
\psi_{4} & =\frac{\ln \left(\frac{S_{t}}{K^{\frac{1}{n}}}\right)+\left(r-D-\frac{\sigma^{2}}{2}\right)\left(T_{2}-T_{1}\right)+n\left(r+\frac{\sigma^{2}}{2}\right)\left(T_{1}-t\right)}{\sigma \sqrt{T_{2}-t}}, \\
\rho & =\frac{\sqrt{T_{1}-t}}{\sqrt{T_{2}-t}} .
\end{aligned}
$$

In this pricing formula, $\Phi$ represents distribution function of standard normal distribution and $\mathbf{\Phi}$ represents the bivariate cumulative distribution function of standard normal distribution and $\rho$ is the correlation.

Proof. Let us begin by explicitly giving the Black-Scholes price of the underlying power option with maturity $T_{2}$ and strike $K_{2}$, with inception at $T_{1}$, can be given as:

$$
C_{\text {pow }}\left(S_{T_{1}}, K_{2}, T_{2}-T_{1}\right)=S_{T_{1}}^{n} e^{\left[(n-1)\left(r+\frac{n \sigma^{2}}{2}\right)-n D\right]\left(T_{2}-T_{1}\right)} \Phi\left(d_{1}\right)-K_{2} e^{-r\left(T_{2}-T-1\right)} \Phi\left(d_{2}\right)
$$

Here

$$
d_{1}=\frac{\ln \left(\frac{S_{T_{1}}}{K^{\frac{1}{n}}}\right)+\left(r-D+\left(n-\frac{1}{2}\right) \sigma^{2}\right)\left(T_{2}-T_{1}\right)}{\sigma \sqrt{T_{2}-T_{1}}}, d_{2}=\frac{\ln \left(\frac{S_{T_{1}}}{K^{\frac{1}{n}}}\right)+\left(r-D-\frac{\sigma^{2}}{2}\right)\left(T_{2}-T_{1}\right)}{\sigma \sqrt{T_{2}-T_{1}}} .
$$

Before moving ahead towards the derivation of the price of compound option contigent upon a power, let us make sense of distribution and dynamics of the process $\left(S_{t}^{n}\right)_{t \geq 0}$ under a risk-neutral measure. From Girsanov's theorem (cf. [22]), under the risk-neutral measure $\mathbb{Q}$ we can write

$$
d S_{t}=(r-D) S_{t} d t+\sigma S_{t} d W_{t}^{\mathbb{Q}}
$$

such that $W_{t}^{Q}=W_{t}+\left(\frac{\mu-r}{\sigma}\right) t$ is a $\mathbb{Q}$-standard Wiener process. By application of Ito's lemma, we may infer

$$
\begin{aligned}
d S_{t}^{n} & =n S_{t}^{n}\left[(r-D) d t+\sigma d W_{t}^{Q}\right]+\frac{n(n-1) \sigma^{2}}{2} S_{t}^{n} d t \\
& =n S_{t}^{n}\left[\left((r-D)-\frac{\sigma^{2}(n-1)}{2}\right) d t+\sigma d W_{t}^{Q}\right]
\end{aligned}
$$


Moreover, application of Ito's lemma on process $\left(\ln \left(S_{t}^{n}\right)\right)_{t \geq 0}$, it follows that,

$$
\begin{aligned}
d \ln \left(S_{t}^{n}\right) & =\left(n(r-D)+\frac{n(n-1) \sigma^{2}}{2}-\frac{n^{2} \sigma^{2}}{2}\right) d t+n \sigma d W_{t}^{\mathbb{Q}} \\
& =\left(n(r-D)-\frac{n \sigma^{2}}{2}\right) d t+n \sigma d W_{t}^{Q} \\
& =n\left(r-D-\frac{\sigma^{2}}{2}\right) d t+n \sigma d W_{t}^{Q}
\end{aligned}
$$

On integrating both sides we may infer,

$$
\begin{aligned}
\int_{t}^{T} d \ln \left(S_{u}^{n}\right) & =\int_{t}^{T} n\left(r-D-\frac{\sigma^{2}}{2}\right) d u+\int_{t}^{T} n \sigma d W_{u}^{Q} \\
\ln \left(\frac{S_{T}^{n}}{S_{t}^{n}}\right) & =n\left(r-D-\frac{\sigma^{2}}{2}\right)(T-t)+n \sigma\left(W_{T}^{Q}-W_{t}^{Q}\right) i . e . \\
S_{T}^{n} & =S_{t}^{n} e^{n\left(r-D-\frac{\sigma^{2}}{2}\right)(T-t)+n \sigma\left(W_{T}^{Q}-W_{t}^{Q}\right)} .
\end{aligned}
$$

As $W_{T}^{\mathbb{Q}}-W_{t}^{\mathbb{Q}}=W_{T-t}^{\mathbb{Q}} \sim \mathcal{N}(0, T-t)$. so it is easy to see that,

$$
\ln \left(\frac{S_{T}^{n}}{S_{t}^{n}}\right) \sim \mathcal{N}\left[n\left(r-D-\frac{\sigma^{2}}{2}\right)(T-t), n^{2} \sigma^{2}(T-t)\right]
$$

We aim to find pricing formula for the price $C_{C_{\text {pow }}}\left(t, S_{t}, K_{1}, K_{2}, T_{1}, T_{2}\right)$ of compound option with maturity $T_{1}$ and Strike $K_{1}$, contingent upon power option whose price mentioned above. Using the Feynman-Kac formula [22, Theorem 4.33]

$C_{C_{\text {pow }}}\left(t, S_{t}, K_{1}, K_{2}, T_{1}, T_{2}\right)$

$$
\begin{aligned}
= & \left.e^{-r\left(T_{1}-t\right)} \mathbb{E}^{\mathbb{Q}}\left[C_{\text {pow }}\left(S_{T_{1}}, K_{2}, T_{2}-T_{1}\right)-K_{1}\right)^{+} \mid \mathcal{F}_{t}\right] \\
= & e^{-r\left(T_{1}-t\right)} \mathbb{E}_{\mathbb{Q}}\left[\left(S_{T_{1}}^{n} e^{\left((n-1)\left(r+\frac{n \sigma^{2}}{2}\right)-n D\right)\left(T_{2}-T_{1}\right)} \Phi\left(d_{1}\right)-K_{2} e^{-r\left(T_{2}-T_{1}\right)} \Phi\left(d_{2}\right)-K_{1}\right)^{+} \mid \mathcal{F}_{t}\right] \\
= & e^{-r\left(T_{1}-t\right)} \int_{0}^{\infty}\left(S_{T_{1}}^{n} e^{\left((n-1)\left(r+\frac{n \sigma^{2}}{2}\right)-n D\right)\left(T_{2}-T_{1}\right)} \Phi\left(d_{1}\right)-K_{2} e^{-r\left(T_{2}-T_{1}\right)} \Phi\left(d_{2}\right)-K_{1}\right)^{+} \\
& \times n\left(S_{T_{1}} \mid S_{t}\right) d S_{T_{1}}
\end{aligned}
$$

Let $S^{*}$ be price of unit of foreign currency such that, the compound option is at the money i.e. $C\left(S^{*}, T_{1} ; K_{2}, T_{2}\right)=K_{1}$, and using this equation and any suitable method (say Newton-Raphson) an approximate value of $S^{*}$ can be computed. Now, 


$$
\begin{aligned}
& C_{C_{\text {pow }}}\left(t, S_{t}, K_{1}, K_{2}, T_{1}, T_{2}\right) \\
&=\quad e^{-r\left(T_{1}-t\right)} \int_{S^{*}}^{\infty}\left(S_{T_{1}}^{n} e^{\left((n-1)\left(r+\frac{n \sigma^{2}}{2}\right)-n D\right)\left(T_{2}-T_{1}\right)} \Phi\left(d_{1}\right)-K_{2} e^{-r\left(T_{2}-T_{1}\right)} \Phi\left(d_{2}\right)-K_{1}\right) \\
& \times n\left(S_{T_{1}} \mid S_{t}\right) d S_{T_{1}}, \\
&= e^{-r\left(T_{1}-t\right)+\left((n-1)\left(r+\frac{n \sigma^{2}}{2}\right)-n D\right)\left(T_{2}-T_{1}\right)} \int_{S^{*}}^{\infty} S_{T_{1}}^{n} \Phi\left(d_{2}\right) n\left(S_{T_{1}} \mid S_{t}\right) d S_{T_{1}} \\
&-e^{-r\left(T_{2}-t\right)} K_{2} \int_{S^{*}}^{\infty} \Phi\left(d_{1}\right) n\left(S_{T_{1}} \mid S_{t}\right) d S_{T_{1}} \\
&-e^{-r\left(T_{1}-t\right)} K_{1} \int_{S^{*}}^{\infty} n\left(S_{T_{1}} \mid S_{t}\right) d S_{T_{1}}=: J_{1}-J_{2}-J_{3} .
\end{aligned}
$$

Let start by computing $J_{1}$ explicitly,

$$
J_{1}=e^{-r\left(T_{1}-t\right)+\left((n-1)\left(r+\frac{n \sigma^{2}}{2}\right)-n D\right)\left(T_{2}-T_{1}\right)} \int_{S^{*}}^{\infty} S_{T_{1}}^{n} \Phi\left(d_{2}\right) n\left(S_{T_{1}} \mid S_{t}\right) d S_{T_{1}},
$$

Now the compound option will be in the money and hence will be exercised iff $S_{T_{1}}^{n}>S^{*}$ iff $S_{t}^{n} e^{n\left(r-D-\frac{\sigma^{2}}{2}\right)\left(T_{1}-t\right)+n \sigma\left(W_{T_{1}}^{Q}-W_{t}^{Q}\right)}>S^{*}$ iff $S_{t}^{n} e^{n\left(r-D-\frac{\sigma^{2}}{2}\right)\left(T_{1}-t\right)+n \sigma \sqrt{T_{1}-t} x}>S^{*}$ iff $x>$ $\frac{\ln \left(\frac{S^{*}}{S_{t}^{n}}\right)-n\left(r-D-\frac{\sigma^{2}}{2}\right)\left(T_{1}-t\right)}{n \sigma \sqrt{T_{1}-t}}=: \psi_{1}$. Hence we rewrite $J_{1}$ as,

$$
\begin{aligned}
J_{1}= & S_{t}^{n} e^{-r\left(T_{1}-t\right)+\left((n-1)\left(r+\frac{n \sigma^{2}}{2}\right)-n D\right)\left(T_{2}-T_{1}\right)+n\left(r-D-\frac{\sigma^{2}}{2}\right)\left(T_{1}-t\right)} \times \\
& \int_{\psi_{1}}^{\infty} \Phi\left(\frac{\ln \left(\frac{S_{t}}{K^{\frac{1}{n}}}\right)+\left(r-D-\frac{\sigma^{2}}{2}\right)\left(T_{2}-T_{1}\right)+n\left(r-\frac{\sigma^{2}}{2}\right)\left(T_{1}-t\right)+n x \sigma \sqrt{T_{1}-t}}{\sigma \sqrt{T_{2}-T_{1}}}\right) \\
& \left.\times \frac{e^{-\frac{\left(x^{2}-2 x \sigma \sqrt{T_{1}-t}\right)}{2}} d x,}{\sqrt{2 \pi}}\right) \\
= & S_{t}^{n} e^{-r\left(T_{1}-t\right)+\left((n-1)\left(r+\frac{n \sigma^{2}}{2}\right)-n D\right)\left(T_{2}-T_{1}\right)+n\left(r-D-\frac{\sigma^{2}}{2}\right)\left(T_{1}-t\right)-\frac{\sigma^{2}}{2}\left(T_{1}-t\right)} \times \\
& \int_{\psi_{1}}^{\infty} \Phi\left(\frac{\ln \left(\frac{S_{t}}{K^{\frac{1}{n}}}\right)+\left(r-D-\frac{\sigma^{2}}{2}\right)\left(T_{2}-T_{1}\right)+n\left(r-D-\frac{\sigma^{2}}{2}\right)\left(T_{1}-t\right)+n x \sigma \sqrt{T_{1}-t}}{\sigma \sqrt{T_{2}-T_{1}}}\right) \\
& \times \frac{e^{-\frac{\left(x-\sigma \sqrt{T_{1}-t}\right)^{2}}{2}}}{\sqrt{2 \pi}} d x .
\end{aligned}
$$

To simplify the above integral, let using substitution $u=-\left(x-\sigma \sqrt{T_{1}-t}\right)$, the integral simplifies to,

$$
\begin{aligned}
J_{1} & =S_{t}^{n} e^{\left((n-1)\left(r+\frac{\sigma^{2}}{2}\right)-n \sigma^{2}\right)\left(T_{1}-t\right)+(n-1)\left(r+\frac{n \sigma^{2}}{2}\right)\left(T_{2}-T_{1}\right)-n D\left(T_{2}-t\right)} \times \\
& \int_{-\infty}^{\psi_{2}} \Phi\left(\frac{\ln \left(\frac{S_{t}}{K^{\frac{1}{n}}}\right)+\left(r-D-\frac{\sigma^{2}}{2}\right)\left(T_{2}-T_{1}\right)+n\left(r-D+\frac{\sigma^{2}}{2}\right)\left(T_{1}-t\right)-n \sigma \sqrt{T_{1}-t} u}{\sigma \sqrt{T_{2}-T_{1}}}\right) \frac{e^{-\frac{u^{2}}{2}}}{\sqrt{2 \pi}} d u
\end{aligned}
$$

where $\psi_{2}=\sigma \sqrt{T_{1}-t}-\psi_{1}$. If we set

$$
\rho=\sqrt{\frac{T_{1}-t}{T_{2}-t}} \text { and } \psi_{4}=\frac{\ln \left(\frac{S_{t}}{K^{\frac{1}{n}}}\right)+\left(r-D-\frac{\sigma^{2}}{2}\right)\left(T_{2}-T_{1}\right)+n\left(r+\frac{\sigma^{2}}{2}\right)\left(T_{1}-t\right)}{\sigma \sqrt{T_{2}-t}}
$$


along with using Bivariate identity (2.1) the last integral can be written as,

$$
\begin{aligned}
J_{1} & =S_{t}^{n} e^{\left((n-1)\left(r+\frac{\sigma^{2}}{2}\right)-n \sigma^{2}\right)\left(T_{1}-t\right)+(n-1)\left(r+\frac{n \sigma^{2}}{2}\right)\left(T_{2}-T_{1}\right)-n D\left(T_{2}-t\right)} \int_{-\infty}^{\psi_{2}} \Phi\left(\frac{\psi_{4}-\rho u}{\sqrt{1-\rho^{2}}}\right) \frac{e^{-\frac{u^{2}}{2}}}{\sqrt{2 \pi}} d u \\
& =S_{t}^{n} e^{\left((n-1)\left(r+\frac{\sigma^{2}}{2}\right)-n \sigma^{2}\right)\left(T_{1}-t\right)+(n-1)\left(r+\frac{n \sigma^{2}}{2}\right)\left(T_{2}-T_{1}\right)-n D\left(T_{2}-t\right)} \mathbf{\Phi}\left(\psi_{2}, \psi_{4} ; \rho\right) .
\end{aligned}
$$

Now let us compute the second integral $J_{2}$,

$$
J_{2}=-e^{-r\left(T_{2}-t\right)} K_{2} \int_{S^{*}}^{\infty} \Phi\left(d_{1}\right) n\left(S_{T_{1}} \mid S_{t}\right) d S_{T_{1}}
$$

By arguing same as previously, the compound option will be in the money and hence will be exercised iff $S_{T_{1}}>S^{*}$ iff $S_{t}^{n} e^{n\left(r-D-\frac{\sigma^{2}}{2}\right)\left(T_{1}-t\right)+n \sigma\left(W_{T_{1}}^{Q}-W_{t}^{Q}\right)}>S^{*}$ iff $S_{t}^{n} e^{n\left(r-D-\frac{\sigma^{2}}{2}\right)\left(T_{1}-t\right)+n \sigma \sqrt{T_{1}-t} x}>S^{*}$ iff $x>$ $-\frac{\ln \left(\frac{S_{t}^{n}}{S^{*}}\right)-\left(r-D-\frac{\sigma^{2}}{2}\right)\left(T_{1}-t\right)}{n \sigma \sqrt{T_{1}-t}}=-\psi_{1}$. Hence we rewrite $J_{2}$ as,

$$
J_{2}=K_{2} e^{-r\left(T_{1}-t\right)} \int_{-\psi_{1}}^{\infty} \Phi\left(\frac{\ln \left(\frac{S_{t}}{K^{\frac{1}{n}}}\right)+\left(r-D+\left(n-\frac{1}{2}\right) \sigma^{2}\right)\left(T_{2}-t\right)+x \sigma \sqrt{T_{1}-t}}{\sigma \sqrt{T_{2}-T_{1}}}\right) \frac{e^{-\frac{x^{2}}{2}}}{\sqrt{2 \pi}} d x
$$

Using the substitution, $u=-x$, the above integral can be simplified to,

$$
\begin{aligned}
J_{2} & =-K_{2} e^{-r\left(T_{1}-t\right)} \int_{\psi_{1}}^{-\infty} \Phi\left(\frac{\ln \left(\frac{S_{t}}{K^{\frac{1}{n}}}\right)+\left(r-D+\left(n-\frac{1}{2}\right) \sigma^{2}\right)\left(T_{2}-t\right)-u \sigma \sqrt{T_{1}-t}}{\sigma \sqrt{T_{2}-T_{1}}}\right) \frac{e^{-\frac{u^{2}}{2}}}{\sqrt{2 \pi}} d u \\
& =K_{2} e^{-r\left(T_{1}-t\right)} \int_{-\infty}^{\psi_{1}} \Phi\left(\frac{\ln \left(\frac{S_{t}}{K^{\frac{1}{n}}}\right)+\left(r-D+\left(n-\frac{1}{2}\right) \sigma^{2}\right)\left(T_{2}-t\right)-u \sigma \sqrt{T_{1}-t}}{\sigma \sqrt{T_{2}-T_{1}}}\right) \frac{e^{-\frac{u^{2}}{2}}}{\sqrt{2 \pi}} d u
\end{aligned}
$$

On setting, if we set $\rho=\sqrt{\frac{T_{1}-t}{T_{2}-t}}$ and $\psi_{3}=\frac{\ln \left(\frac{S_{t}}{K^{\frac{1}{n}}}\right)+\left(r-D+\left(n-\frac{1}{2}\right) \sigma^{2}\right)\left(T_{2}-t\right)}{\sigma \sqrt{T_{2}-t}}$ along with using Bivariate identity (2.1) the last integral can be written as,

$$
\begin{aligned}
J_{2} & =K_{2} e^{-r\left(T_{1}-t\right)} \int_{-\infty}^{\psi_{1}} \Phi\left(\frac{\psi_{3}-\rho u}{\sqrt{1-\rho^{2}}}\right) \frac{e^{-\frac{u^{2}}{2}}}{\sqrt{2 \pi}} d u \\
& =K_{2} e^{-r\left(T_{1}-t\right)} \Phi\left(\psi_{1}, \psi_{3} ; \rho\right) .
\end{aligned}
$$


Finally, its fairly easy to compute the 3 rd integral $I_{3}$, we will make use of fact $W_{T_{1}}^{Q}-W_{t}^{Q} \sim \mathcal{N}\left(0, T_{1}-t\right)$ we have

$$
\begin{aligned}
J_{3} & =K_{1} e^{-r\left(T_{1}-t\right)} \int_{S^{*}}^{\infty} n\left(S_{T_{1}} \mid S_{t}\right) d S_{T_{1}} \\
& =K_{1} e^{-r\left(T_{1}-t\right)} \mathbb{Q}\left(S_{T_{1}}>S^{*} \mid S_{t}\right) \\
& =K_{1} e^{-r\left(T_{1}-t\right)} \mathbb{Q}\left(S_{t}^{n} e^{n\left(r-D-\frac{\sigma^{2}}{2}\right)\left(T_{1}-t\right)+n \sigma\left(W_{T_{1}}^{Q}-W_{t}^{Q}\right)}>S^{*} \mid S_{t}\right) \\
& =K_{1} e^{-r\left(T_{1}-t\right)} \mathbb{Q}\left(S_{t}^{n} e^{n\left(r-D-\frac{\sigma^{2}}{2}\right)\left(T_{1}-t\right)+n \sigma \sqrt{T_{1}-t} Z}>S^{*} \mid S_{t}\right) \\
& =K_{1} e^{-r\left(T_{1}-t\right)} \mathbb{Q}\left(Z>\frac{\ln \left(\frac{S^{*}}{S_{t}^{n}}\right)-n\left(r-D-\frac{\sigma^{2}}{2}\right)\left(T_{1}-t\right)}{n \sigma \sqrt{T_{1}-t}}\right) \\
& =K_{1} e^{-r\left(T_{1}-t\right)} \Phi\left(\psi_{1}\right) .
\end{aligned}
$$

Combine the integrals $J_{1}, J_{2}$ and $J_{3}$ in $(2.2)$ we get the pricing formula for European compound Option written on Foreign Currency call option,

$$
\begin{aligned}
C_{C_{\text {pow }}}\left(t, S_{t}, K_{1}, K_{2}, T_{1}, T_{2}\right)= & S_{t}^{n} e^{\left((n-1)\left(r+\frac{\sigma^{2}}{2}\right)-n \sigma^{2}\right)\left(T_{1}-t\right)+(n-1)\left(r+\frac{n \sigma^{2}}{2}\right)\left(T_{2}-T_{1}\right)-n D\left(T_{2}-t\right)} \\
& \times \mathbf{\Phi}\left(\psi_{2}, \psi_{4} ; \rho\right)-K_{2} e^{-r\left(T_{1}-t\right)} \boldsymbol{\Phi}\left(\psi_{1}, \psi_{3} ; \rho\right)-K_{1} e^{-r\left(T_{1}-t\right)} \Phi\left(\psi_{1}\right) .
\end{aligned}
$$

where

$$
\begin{aligned}
\psi_{1} & =\frac{\ln \left(\frac{S^{*}}{S_{t}^{n}}\right)-n\left(r-D-\frac{\sigma^{2}}{2}\right)\left(T_{1}-t\right)}{\sigma \sqrt{T_{1}-t}}, \\
\psi_{2} & =\frac{\ln \left(\frac{S_{t}^{n}}{S^{*}}\right)+n\left(r-D+\frac{\sigma^{2}}{2}\right)\left(T_{1}-t\right)}{n \sigma \sqrt{T_{1}-t}}, \\
\psi_{3} & =\frac{\ln \left(\frac{S_{t}}{K^{\frac{1}{n}}}\right)+\left(r-D+\left(n-\frac{1}{2}\right) \sigma^{2}\right)\left(T_{2}-t\right)}{n \sigma \sqrt{T_{2}-t}}, \\
\psi_{4} & =\frac{\ln \left(\frac{S_{t}}{K^{\frac{1}{n}}}\right)+\left(r-D-\frac{\sigma^{2}}{2}\right)\left(T_{2}-T_{1}\right)+n\left(r+\frac{\sigma^{2}}{2}\right)\left(T_{1}-t\right)}{\sigma \sqrt{T_{2}-t}}, \\
\rho & =\frac{\sqrt{T_{1}-t}}{\sqrt{T_{2}-t}} .
\end{aligned}
$$

In this pricing formula, $\Phi$ represents cumulative function of standard normal distribution and $\mathbf{\Phi}$ represents the bivariate cumulative normal distribution and $\rho$ is the correlation.

Conflicts of Interest: The author(s) declare that there are no conflicts of interest regarding the publication of this paper.

\section{REFERENCES}

[1] Z. Brzezniak, T. Zastawniak, Basic stochastic processes: a course through exercises. Springer Science \& Business Media. 2000. 
[2] F. Black, M. Scholes, The pricing of options and corporate liabilities. J. Polit. Econ. 81(3)(1973), 637-654.

[3] S. Macovschi, F. Quittard-Pinon, On the pricing of power and other polynomial options. J. Deriv. 13(4)(2006), 61-71.

[4] Z. Zhang, W. Liu, Y. Sheng, Valuation of power option for uncertain financial market. Appl. Math. Comput. 286(2016), $257-264$

[5] E. Dastranj, R. Latifi, A comparison of option pricing models, Int. J. Financ. Eng. 4(02n03)(2017), 1750024.

[6] E. Saberi, S.R. Hejazi, E. Dastranj, A new method for option pricing via time fractional pde, Asian-Eur. J. Math. 11 (5) (2018) 1850074.

[7] E. Dastranj, H.S. Fard, A. Abdolbaghi, S.R. Hejazi, Power option pricing under the unstable conditions (Evidence of power option pricing under fractional Heston model in the Iran gold market). Physica A. Stat. Mech. Appl. 537(2020), 122690.

[8] R. Geske, The valuation of corporate liabilities as compound options. J. Financ. Quant. Anal. 12(1977), 541-552.

[9] R. Geske, The valuation of compound options. J. Financ. Econ. 7(1)(1979), 63-81.

[10] J.C. Hull, Options futures and other derivatives. Pearson Education India. 2003.

[11] A. Elettra, A. Rossella, A generalization of the Geske formula for compound options. Math. Soc. Sci. 45(1)(2003), 75-82.

[12] P. Carr, The valuation of sequential exchange opportunities. J. Finance, 43(5)(1988), 1235-1256.

[13] C. Chiarella, B. Kang, The evaluation of American compound option prices under stochastic volatility and stochastic interest rates. J. Comput. Finance. 17(2013), 71-92.

[14] C. Li, H. Liu, M. Wang, W. Li, The pricing of compound option under variance gamma process by FFT, Commun. Stat., Theory Meth. (2020) 1-15. https://doi.org/10.1080/03610926.2020.1740268.

[15] G. Feiger, B. Jacquillat, Currency Option Bonds, Puts and Calls on Spot Exchange and the Hedging of Contingent Foreign Earnings, J. Finance. 34(1979), 1129-1139.

[16] J.O. Grabbe, The pricing of call and put options on foreign exchange. J. Int. Money Finance, 2(3)(1983), $239-253$.

[17] K.I. Amin, R.A. Jarrow, Pricing foreign currency options under stochastic interest rates. J. Int. Money Finance, 10(3)(1991), $310-329$

[18] J.H. Guo, M.W. Hung, Pricing American options on foreign currency with stochastic volatility, jumps, and stochastic interest rates. J. Futures Mark., Fut. Opt. Other Deriv. Prod. 27(9)(2007), 867-891.

[19] S. Li, J. Peng, B. Zhang, The uncertain premium principle based on the distortion function. Insurance, Math. Econ. $53(2)(2013), 317-324$.

[20] D. Liu, Markov modulated jump-diffusions for currency options when regime switching risk is priced. Int. J. Financ. Eng. 6(04)(2019), 1950038.

[21] M.C. Chuang, C.H. Wen, S.K. Lin, Valuation and empirical analysis of currency options. Int. Rev. Econ. Finance, 66(2020),71-91.

[22] M. Capi'nski, E. Kopp, J. Traple, Stochastic calculus for finance, Cambridge University Press. 2012.

[23] E. Chin, S. Ólafsson, D. Nel, Problems and Solutions in Mathematical Finance: Stochastic Calculus, Vol. 1, The Wiley Finance Series. 2014. 